LAWRENCE LIVERMORE NATIONAL LABORATORY
Magnetic Properties of Ultra Thin Epitaxial Fe

Films on $\mathrm{GaAs}(001)$

M. Spangenberg, J.R. Neal, T.-H. Shen, S.A. Morton, J.G. Tobin, G.D. Waddill, J.A.D. Matthew, D. Greig, A.E.R. Malins, E.A. Seddon, M. Hopkinson

October 2, 2003 
This document was prepared as an account of work sponsored by an agency of the United States Government. Neither the United States Government nor the University of California nor any of their employees, makes any warranty, express or implied, or assumes any legal liability or responsibility for the accuracy, completeness, or usefulness of any information, apparatus, product, or process disclosed, or represents that its use would not infringe privately owned rights. Reference herein to any specific commercial product, process, or service by trade name, trademark, manufacturer, or otherwise, does not necessarily constitute or imply its endorsement, recommendation, or favoring by the United States Government or the University of California. The views and opinions of authors expressed herein do not necessarily state or reflect those of the United States Government or the University of California, and shall not be used for advertising or product endorsement purposes.

This work was performed under the auspices of the U.S. Department of Energy by the University of California, Lawrence Livermore National Laboratory under Contract W7405-Eng-48. 


\title{
Magnetic properties of ultra thin epitaxial Fe films on GaAs(001)
}

\author{
M. Spangenberg, J. R. Neal, T.-H. Shen * \\ Joule Physics Laboratory, Institute for Materials Research, University of Salford, \\ Salford M5 4WT, U.K. \\ S.A. Morton, J.G. Tobin \\ Department of Chemistry and Materials Science, Lawrence Livermore National \\ Laboratory, California 94550, U.S.A. \\ G.D. Waddill \\ Department of Physics, University of \\ Missouri-Rolla, Rolla, MO 65409, U.S.A. \\ J.A.D. Matthew \\ Department of Physics, University of York, York YO1 5DD, U.K. \\ D.Greig \\ Department of Physics and Astronomy, University of Leeds, Leeds LS2 9JT, U.K. \\ A.E.R. Malins, E.A.Seddon \\ CLRC Daresbury Laboratory, Warrington WA4 4AD, U.K. \\ M. Hopkinson \\ EPSRC National Centre for III-V Technologies, University of Sheffield, Mappin \\ Street, Sheffield, S1 3JD, UK
}

\begin{abstract}
The magnetic properties of epitaxial Fe films on GaAs in the range of the first few monolayers have been the subject of a considerable number of investigations in recent years. The absence of magnetic signatures at room temperature has been
\end{abstract}


attributed to the existence of a magnetic 'dead' layer as well as superparamagnetism. By examining the temperature dependence of the magnetic linear dichroism of the $\mathrm{Fe}$ core level photoelectrons, we found that these 'non-ferromagnetic' layers had in fact a Curie temperature, $T_{c}$, substantially lower than room temperature, e.g., a $T_{c}$ of about $240 \mathrm{~K}$ for thin films of a nominal thickness of $0.9 \mathrm{~nm}$. The values of Curie temperature were sensitive to the initial GaAs substrate conditions and the thickness of the Fe over-layer with a layer of thickness of $1.25 \mathrm{~nm}$ showing a $\mathrm{T}_{\mathrm{c}}$ above room temperature. The data suggest that the ultrathin Fe films on $\mathrm{GaAs}(001)$ are ferromagnetic, although a weaker exchange interaction in the films lead to a substantial reduction in Curie temperature.

PACS: 75.70.-i; 75.50.Bb; 78.20.Ls, 79.60.-i; 75.30.Kz 
Current studies of ultra thin magnetic films on semiconductor substrates are largely motivated by possible applications in spin injection devices [1,2,3,4]. Recent demonstrations of spin-coherent transport over large distances in n-type semiconductors [5] and the persistence of spin coherence over a sizeable time scale [6], albeit at a low temperature, are clearly very encouraging. Since the early work of Waldrop and co-workers [7] there have been intensive investigations on the characteristics of Fe thin films on GaAs substrates, as a typical epitaxial magnetic metal- semiconductor system. A variety of different features has been revealed: the interfacial magnetism and its dependence on the intermixing of $\mathrm{Fe}, \mathrm{Ga}$ and $\mathrm{As}$ on the one hand and on the morphology of the deposited Fe on the other hand. Of relevance to the present work, we note that it was proposed that As diffusion into the Fe layer and the formation of non-ferromagnetic $\mathrm{FeAs}_{2}$ like compounds resulted in a magnetically dead interfacial region; above a critical thickness the magnetisation of the Fe layer would depend exponentially on the distance to the interface, correlated with the diffusion of As [8]. Similarly, interdiffusion and compound formation have been highlighted in recent studies by Filipe et al [9]. The exponential increase in the magnetisation observed was interpreted as an intermediate phase of ternary pseudocubic hexagonal FeGaAs, sandwiched between magnetically dead Fe at the interface and pure bulk-like Fe at a thickness of about $20 \mathrm{~nm}$. More recently, in a study using the technique of magneto optical Kerr effect (MOKE), the delayed onset of ferromagnetic behaviour in ultra thin Fe was attributed to Fe clusters forming a superparamagnetic phase which became ferromagnetic as the clusters coalesce at higher Fe coverage [10]. Furthermore, a temperature-dependent measurement of magneto resistance of the ultrathin Fe films was attributed to the existence of such a superparamagnetic phase [11]. 
Whilst recognising that the magnetic behaviour of ultrathin Fe layers is also sensitive to both GaAs substrate surface morphology [12] and surface stoichiometry [13], this Letter reports on the temperature dependent magnetic state of $\mathrm{Fe}$ on $\operatorname{GaAs}(001)$ through a study of the magnetic dichroism of the Fe $2 p$ and $3 p$ core level photoelectrons with linearly polarised radiation.

In recent years X-ray magnetic linear dichroism (XMLD) has been well established as a surface-sensitive, element specific probe of sample magnetisation $[14,15,16]$, which is highly suitable for the study of magnetic properties of ultrathin films of a few atomic layers. Although experimentally a relatively straight-forward measurement, the theory of XMLD is complex and for quantitative analysis a simple and effective model has been developed [16], in the case of $3 p$ sates, which has established a proportional relationship between linear dichroism asymmetry and the core-level exchange splitting, which in turn is assumed to be directly dependent on the valence state exchange splitting and the magnetic moment in the plane of the surface[17]. Therefore it is sufficient in the present study to consider the dichroism asymmetry as a direct measure of the magnetisation of the specimens.

Experiments were carried out with base pressure better than $3 \times 10^{-10} \mathrm{mbar}$ in the Spin Chamber on Beamline 7.0 at the Advanced Light Source, Berkeley $[18,19]$. The Fe/GaAs samples were prepared in situ for the dichroism study, using As capped n-type GaAs epilayers grown on $n^{+}$singular and vicinal GaAs(001) substrates. The As capping layers were removed by thermal desorption prior to Fe deposition. For the clean substrates used in this study, a value of Ga to As ratio of about 1.06 was obtained. In a similar experimental configuration as that previously reported [16], dichroism spectra of the $\mathrm{Fe} 2 \mathrm{p}$ and $\mathrm{Fe} 3 \mathrm{p}$ states were taken using p polarised $45^{\circ}$ incidence photons and normally emitted photoelectrons. A magnetising field was 
applied perpendicular to the incidence plane of the light and the surface normal with the [110] direction of the sample orientated parallel to the field direction. The data were taken with the sample in a remnant magnetisation state.

As an example, Figure 1 shows a typical dichroism result of a $2.7 \mathrm{~nm}$ thick $\mathrm{Fe}$ film on singular GaAs (001) substrate obtained with $810 \mathrm{eV}$ photons. The upper part of Figure 1 displays the raw data of the two Fe $2 p$ core level spectra acquired with the Fe film in opposite remnant states along the easy axis in the [110] direction. Both core levels, the $\mathrm{Fe} 2 \mathrm{p}_{1 / 2}$ and the $\mathrm{Fe} 2 \mathrm{p}_{3 / 2}$, exhibit dichroic features.

The magnetic asymmetry, $A_{M}$, of the core level spectra can be calculated using:

$$
A_{M}=\frac{I^{\uparrow}-I^{\downarrow}}{I^{\uparrow}+I^{\downarrow}}
$$

where $I^{\uparrow}$ and $I^{\downarrow}$ are the measured X-ray photoelectron spectroscopy (XPS) intensities, with Shirley background [20] removed, for the sample magnetized parallel, $\uparrow$, and antiparallel, $\downarrow$, to the [110] direction respectively.

The lower part of Figure 1 shows the asymmetry spectrum of the two opposite remnant states. The overall features of the spectrum agree well with those of the Fe $2 p$ dichroism results in the literature obtained in a similar experimental geometry. For example the spectral features are similar to those observed previously by Rose et al [21] and Waddill et al [22]. Furthermore, we have found that Fe 3p dichroism results of the same sample (shown in Figure 2) are of very similar magnitude. The Fe $3 p$ states have been used for the temperature dependent study as the proportional relationship between the XMLD asymmetry and magnetisation has been previously tested for the $3 p$ states[16]. 
In Figure 3, the peak-to-peak magnetic asymmetries ('total asymmetry'), A тот as indicated in Figure 2, obtained from $\mathrm{Fe} 3 \mathrm{p}$ photoelectrons are displayed as a function of temperature for $0.9 \mathrm{~nm}$ Fe grown on a singular (unfilled circles) and a vicinal (filled circles) GaAs(001) substrate. The vicinal substrate has an off-cut of 3 degree towards the [110] direction. Both samples were grown side-by-side for better comparison and the data taken alternatively as the samples were being warmed up slowly. The results clearly show that both $0.9 \mathrm{~nm}$ Fe films are ferromagnetic with a similar Curie temperature of about $240 \mathrm{~K}$. It is also interesting to note that, the value of the magnetic asymmetry for the Fe on the vicinal substrate is about $8 \%$ higher in the temperature range studied, indicating a somewhat higher magnetisation at the same temperature. This appears to be consistent with our earlier work [12] that, in the ultrathin region, the magnetic characteristics appeared to be enhanced for Fe grown on vicinal substrates.

The value of the Curie temperature has also been found to be very sensitive to surface chemistry as well as surface morphology. For example, we have found that poorly de-capped or contaminated substrates lead to the Fe overlayers having substantially lower values of Curie temperature.

As a technique for probing magnetisation, XMLD would enable us to investigate the temperature dependent remnant states of these ultrathin films thereby gaining further understanding of their magnetic characteristics. However for the present set of results, both the statistical quality of the data and the unavailability of temperatures below $180 \mathrm{~K}$ have limited a more quantitative analysis. Nevertheless, to illustrate the potential of temperature dependent XMLD studies, we shall compare our results with a Weiss-type theoretical model [23]. In the inset of Figure 3, we have reproduced the theoretical curves of normalised spontaneous magnetisation as a 
function of reduced temperature [23]. For clarity, the experimental data of bulk Fe and $\mathrm{Ni}$ in the original diagram have not been included. Due to our limited temperature range, the information on the zero temperature magnetisation, $\mathrm{M}_{0}$, is lacking. Unfortunately, fitting to the different values of total momentum quantum number, $\mathrm{J}$, is sensitive to $\mathrm{M}_{0}$. However we note that for thicker films the magnetic properties as measured by spin polarised photoelectron spectroscopy displayed bulk-like behaviour [13] and the values of Curie temperature are substantially higher than room temperature (please refer to Figure 4 and later discussions). We assume it to be a sensible estimation to use the magnetic asymmetry of $10 \mathrm{~nm}$ Fe on a vicinal substrate (about $16 \%$ ) as a measure of $\mathrm{M}_{0}$ for the $0.9 \mathrm{~nm} \mathrm{Fe}$ on the vicinal substrate. We also take into account the $8 \%$ difference between the films on vicinal and singular substrate for the normalisation of the singular results. The normalised data, as an approximate measure of the normalised spontaneous magnetisation, are then superimposed to the inset of Figure 3. It appears that our data are clustered close to the $\mathrm{J}=1 / 2$ curve, just like that of bulk $\mathrm{Fe}$ and $\mathrm{Ni}$, suggesting that the electron spin is likely to be the main contribution to the atomic moment. The larger deviation as the temperature approaches to the transition temperature is usually accounted for as the failure of the theory close to the critical point. We wish to emphasise that the above discussion depends sensitively on the estimation of $\mathrm{M}_{0}$. To fit to the $\mathrm{J}=8$ curve, a value of the magnetic asymmetry of about $25 \%$ would be required.

We shall now discuss the sensitivity of the Curie temperature with regard to additional Fe coverage. In Figure 4, the XMLD data taken close to room temperature are presented for the $0.9 \mathrm{~nm} \mathrm{Fe}$ on singular GaAs (Figure 3(a), 283K) and that for a further deposition of $0.35 \mathrm{~nm} \mathrm{Fe}$ (Figure 3(b), 298K). Whilst the former showed an essentially zero magnetic asymmetry, the latter displayed a non-zero value. It is clear 
from the results that the additional coverage of $0.35 \mathrm{~nm}$ of $\mathrm{Fe}$ has enhanced the magnetic interaction between the Fe atoms, resulting an increase of the Curie point to well above room temperature.

Our measurements suggest that in the initial stage of $\mathrm{Fe}$ coverage, the magnetic interaction has been weakened by inter-diffusion as well as islanding. Consequently the Curie temperature has been reduced to well below room temperature. There seems to be a critical Fe coverage, likely to correspond to a reasonable degree of coalescence of islands, above which the films become ferromagnetic at room temperature. However it does not seem to be clear that the nonferromagnetic phase is of superparamagnetic in nature, especially considering the close proximity of the $\mathrm{Fe}$ islands and the relatively long range of the exchange interaction.

To summarise, we have carried out a temperature dependent study of the XMLD of ultrathin Fe films on singular and vicinal GaAs(001) substrates. Values of Curie temperature well below room tempe rature have been found, e.g., $240 \mathrm{~K}$ for a 0.9 nm Fe on $\mathrm{GaAs}(001)$, but the values are highly sensitive to the stoichiometry and morphology of substrate surfaces as well as Fe overlayer coverage. We believe that this work demonstrates that as a surface-sensitive, element-specific probe of sample magnetisation, XMLD and its temperature dependent behaviours have tremendous potential in the study of magnetic characteristics of ultrathin films and related systems. Further work is planned to enable a more quantitative investigation.

\section{Acknowledgement}

We acknowledge gratefully the support by the UK Engineering and Physical Sciences Research Council (EPSRC) and a travel grant by the University of Salford. 
Both MS and JRN would like to thank the EPSRC for their research studentship. This work was performed under the auspices of the U.S. DOE by UC Lawrence Livermore National Laboratory under contract W-7405-Eng-48. Experiments were carried out at the Spectromicroscopy Facility (Beamline 7.0) at the Advanced Light Source, built and supported by the U.S. Department of Energy. 


\section{Reference:}

[1] I. Malajovich, J.J. Berry, N. Samarth, D.D. Awschalom: Nature 411, 770 (2001)

[2] Y. Ohno, D.K. Young, B. Beschoten, F. Matsukura, H. Ohno, D.D. Awschalom: Nature 402, 790 (1999)

[3] A.T. Filip. B.H. Hoving, F.J. Jedema, B.J. van Wees, B. Dutta, S. Borghs: Phys. Rev. B 62, 9996 (2000)

[4] P.R. Hammar, B.R.Bennett, M.J. Yang, M. Johnson: Phys. Rev. Lett. 83, 203 (1999)

[5] J.M. Kikkawa, D.D. Awschalom: Nature 397, 139 (1999)

[6] J.M. Kikkawa, D.D. Awschalom: Phys. Rev. Lett. 80, 4313 (1998)

[7] J.R. Waldrop and R.W. Grant, Appl. Phys. Lett. 34, 630 (1979)

[8] G.A. Prinz, in Ultrathin Magnetic Structures edited by B. Heinrich and J.A.C. Bland (Spinger-Verlag, Berlin, 1994) Vol.2, pp 1-44

[9] A. Filipe, A. Schuhl, P. Galtier, Appl. Phys. Lett. 70, 129 (1997); A. Filipe and A. Shuhl, J. Appl. Phys. 81, 4359 (1997)

[10] Y.B. Xu, E.T. Kernohan, D.J. Freeland, A. Ercole, M. Tselepi and J.A.C. Bland, Phys. Rev. B 58, 890 (1998)

[11] C.M. Gurtler, Y.B. Xu, J.A.C. Bland, J. M.M.M. 226, 655 (2001)

[12] T.Zhang, M.Spangenberg, D.Greig, N.Takahashi, T-H,Shen, J.A.D.Matthew, S.Comelius, M.Rendall, E.A.Seddon, Appl. Phys. Lett. 78, 961 (2001)

[13] N. Takahashi, T. Zhang, M. Spangenberg, D. Greig, T.H. Shen, S. Cornelius, E.A. Seddon and J.A.D. Matthew, Surf. Rev. and Lett. 9, 693 (2002)

[14] F.O. Schmann, R.F. Willis, K.G. Goodman and J.G. Tobin, Phys. Rev. Lett. 79, $5166(1997)$

[15] F.O. Schmann, R.F. Willis, and J.G. Tobin, J.V.S.T. A18, 1259 (2000) 
[16] J.G. Tobin and F.O. Schumann, Surf. Sci. 478, 211(2001)

[17] F.J. Himpsel, Phys. Rev. Lett. 67, 2363 (1991)

[18] M. Hochstrasser, J.G. Tobin, E. Rotenberg and S.D. Kevan, Phys. Rev. Lett. 89, $216802(2002)$

[19] S.A. Morton, G.D. Waddill, S. Kim, I.K. Schuller, S.A. Chambers, and J.G. Tobin, Surf. Sc. Lett. 513, L451 (2002)

[20] D.A. Shirley, Phys. Rev. B, 5, 4709 (1972)

[21] H.B. Rose, T. Kinoshita, C. Roth and F.U. Hillebrect, in Spin-orbit-Influenced Spectroscopies of Magnetic Solids, edited by G. Schutz and H. Ebert (Springer, Berlin, 1996) p105

[22] G.D. Waddill, J.G. Tobin and D.P. Pappas, Phys. Rev. B, Rapid Comm. 46, 552 (1992)

[23] F. Tyler, Phil. Mag., 11, 596 (1931) 


\section{Caption:}

Figure 1. Dichroism in Fe $2 \mathrm{p}$ core level photoemission of $2.7 \mathrm{~nm}$ thick Fe film on singular GaAs(001) substrate, as excited by $810 \mathrm{eV}$ plinearly polarised photons. 'Mag up' and 'Mag down' indicate the two remnant states parallel and antiparallel to [110] direction.

Figure 2. Dichroism in Fe $3 p$ core level photoemission of $2.7 \mathrm{~mm}$ thick Fe film on singular GaAs(001) substrate, with $160 \mathrm{eV}$ p-linearly polarised photons. The meaning of the 'Mag up' and 'Mag down' notations is the same as in Figure 1. A $_{\text {TOT }}$ denotes the peak-to-peak asymmetry.

Figure 3. A temperature dependence of the peak-to-peak magnetic asymmetry ('Total Asymmetry') of Fe 3p photoelectrons for $0.9 \mathrm{~nm}$ Fe films on GaAs (001) substrates. The inset shows a likely fit to the temperature dependent spontaneous magnetisation as predicted by the Weiss-type theory [23].

Figure 4. Dichroism measurements of Fe $3 p$ close to room temperature on (a) $0.9 \mathrm{~nm}$ Fe on singular GaAs (001) and (b) followed by an additional $0.35 \mathrm{~nm}$ Fe deposition. The meaning of the 'Mag up' and 'Mag down' notations is the same as in Figure 1. 


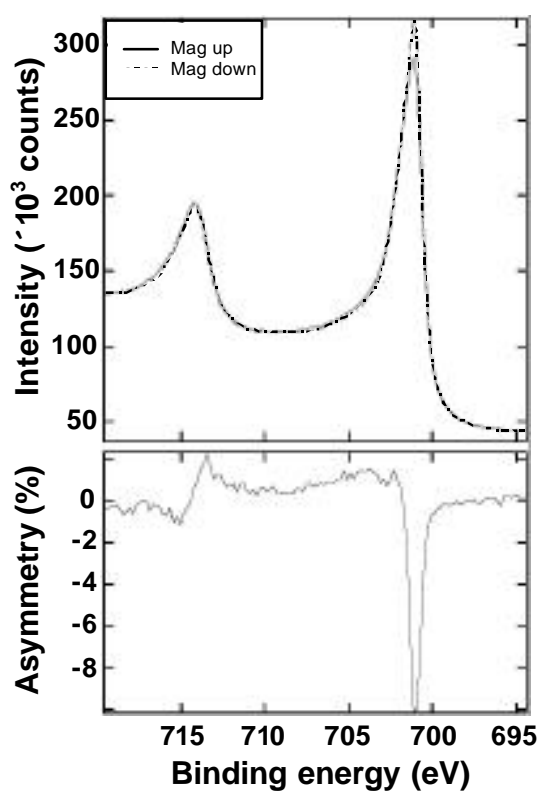

Figure 1 


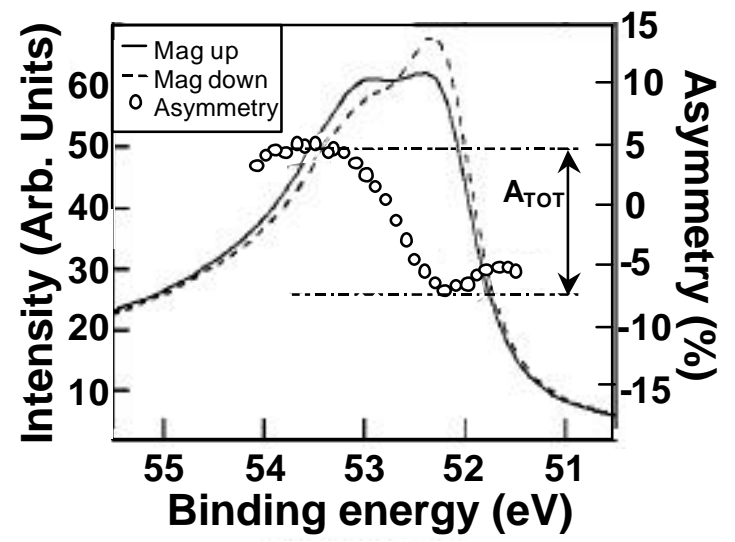

Figure 2 


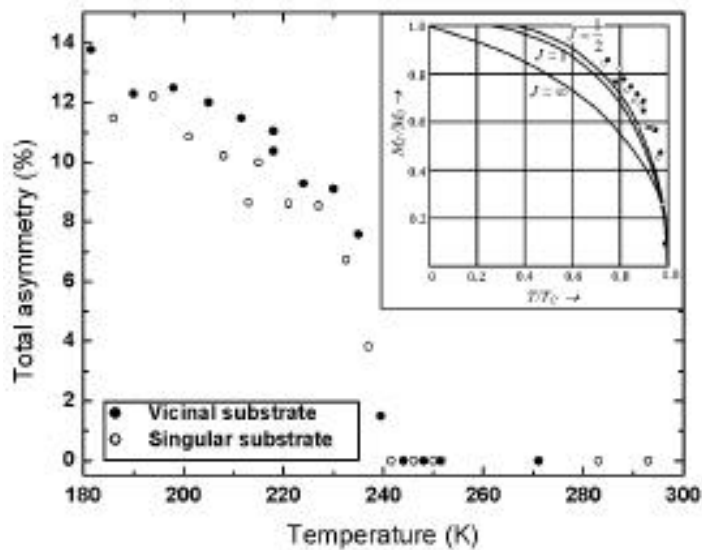

Figure 3 
(a)

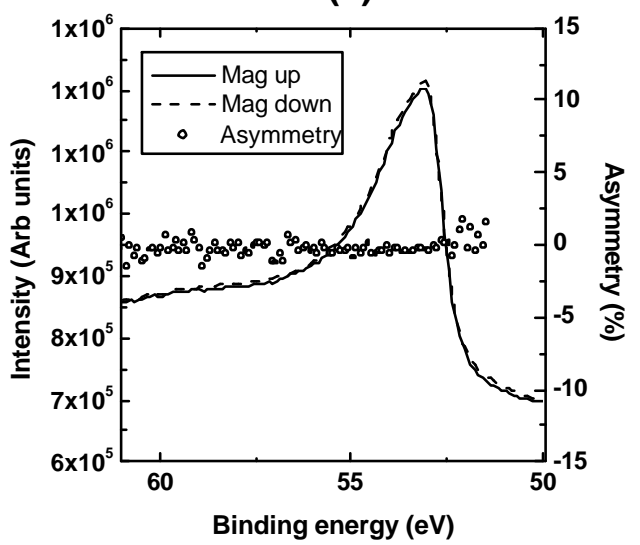

(b)

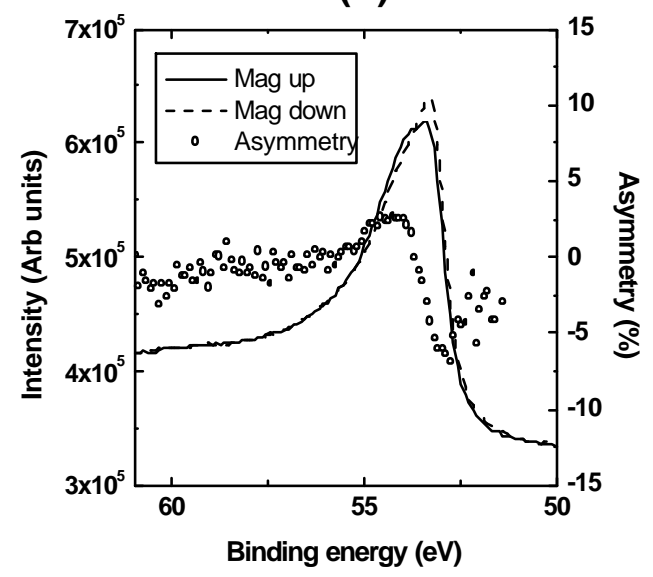

Figure 4 